\title{
Parameter space mapping of InAs nanowire crystal structure
}

\author{
Kimberly A. Dick ${ }^{\text {a) }}$ \\ Solid State Physics, Lund University, Box 118, S-221 00 Lund, Sweden and Polymer and Materials \\ Chemistry, Lund University, Box 124, S-221 00 Lund, Sweden \\ Jessica Bolinsson, Maria E. Messing, Sebastian Lehmann, and Jonas Johansson \\ Solid State Physics, Lund University, Box 118, S-221 00 Lund, Sweden \\ Philippe Caroff \\ Institut d'Électronique, de Microélectronique et de Nanotechnologie, UMR CNRS 8520, Avenue Poincaré, \\ BP 60069, 59652 Villeneuve d'Ascq, France
}

(Received 26 February 2011; accepted 26 April 2011; published 3 June 2011)

\begin{abstract}
Crystal structure and defects have been shown to have a strong impact on III-V nanowire properties. Recently, it was demonstrated that the issue of random stacking and polytypism in semiconductor nanowires can often be controlled using accessible growth parameters (such as temperature, diameter, and V/III ratio). In addition, it has been shown that crystal phase can be tuned selectively between cubic zinc blende and hexagonal wurtzite within individual nanowires of III-V materials such as InAs. In order for such results to be generally applied to different growth setups, it is necessary to fully explore and understand the trends governing crystal phase dependencies on all accessible growth parameters, including how they relate to each other. In this study, the authors have systematically investigated the influence of temperature, diameter, V/III ratio, and total mass flow on the crystal structure of InAs nanowires grown by metal-organic vapor phase epitaxy over a broad parameter range. The authors observed that each of these accessible parameters can affect the resulting crystal structure, and that the trends for each parameter are affected by the magnitude of the others. The authors also noted that most of the parameter dependencies are nonlinear and, in fact, exhibit threshold values at which structure changes discontinuously. By optimizing each of the growth parameters, it is shown that pure $\mathrm{ZB}$ or pure $\mathrm{WZ}$ phase can be achieved for several different sets of growth conditions. The roles of nucleation kinetics, thermodynamics, and precursor chemistry are also discussed to compare the results to current nanowire growth models. The results in this work should facilitate comparison of data and transfer of knowledge between different growth systems and techniques, which, in turn, should lead to greater understanding of polytypism in nanowires and greater control and freedom in nanowire crystal phase engineering. (C) 2011 American Vacuum Society. [DOI: 10.1116/1.3593457]
\end{abstract}

\section{INTRODUCTION}

Crystal quality has always been of utmost importance for reliability and performance of III-V semiconductor devices. Expectations on crystal quality are even higher when reaching for the nanoscale range because single defects or impurities can dramatically affect nanodevice properties. ${ }^{1,2}$ III-V nanowires grown by standard epitaxy techniques have anticipated applications in the fields of electronics, optics, and sensing applications. Unfortunately, however, the majority of grown III-V nanowires naturally contains uncontrolled mixtures of polytypes and/or randomly arranged stacking defects (such as stacking faults or twin planes). Since the different polytypes can have different electronic band structures, ${ }^{3-5}$ and interruptions in the stacking sequence can also act as scattering centers for electrons, ${ }^{6,7}$ uncontrolled crystal structure is challenging for device applications. In recent years, this has triggered extensive experimental efforts directed toward improving the crystal quality of III-V nanowires.

On the other hand, the polytypism inherent to III-V nanowires may present unique opportunities. Most III-V materials

${ }^{a)}$ Electronic mail: kimberly.dick@ftf.lth.se can exhibit both the cubic zinc blende (ZB) phase (stable for most bulk III-Vs) and the hexagonal wurtzite (WZ) phase (stable for III-N materials) in nanowires, and even higher polytypes such as $4 H$ and $6 H$ have been reported. ${ }^{8,9}$ Semiconductor nanowires thus offer an extra degree of freedom for design of advanced crystal phase engineered nanowirebased devices compared to their standard two-dimensional counterparts. Among III-V materials, InAs has shown particular promise for crystal phase engineering and control, ${ }^{10,11}$ while its high electron mobility and low contact resistance make it interesting for electronic applications.

There are many investigations of the effect of growth parameters on the crystal structure of III-V nanowires (for a comprehensive review, see Ref. 12). Most investigations focus solely on one growth parameter, such as temperature, ${ }^{13,14}$ precursor flows,${ }^{15}$ precursor V/III ratio, ${ }^{15}$ diameter, ${ }^{17}$ growth time, ${ }^{18}$ or impurity atoms. ${ }^{19}$ Interestingly, there are no reports of nanowire crystal structure being tuned from $100 \%$ pure $\mathrm{ZB}$, through a mixture of structures, to $100 \%$ pure $\mathrm{WZ}$ using only one experimental parameter. Therefore, several studies have investigated two parameters together and attempted to correlate these to achieve greater structural freedom. ${ }^{11,20-22}$ 
In these cases, it is clear that the dependence of the crystal structure on one parameter depends on the magnitude of the second parameter. It is therefore inferred that a full understanding of the crystal structure requires mapping of all relevant parameters against each other. Without this, it is not possible to directly compare reports of crystal structure variations, which are performed in different growth systems, inevitably with at least one growth parameter differing.

Comparison of crystal structure investigations in literature illustrates this point very clearly. Taking InAs nanowires seeded by Au particles as just one example, the investigation by Caroff et al. shows that the structure changes from WZdominant at lower temperatures to more $\mathrm{ZB}$-containing at higher temperatures for the temperature range of $420-480{ }^{\circ} \mathrm{C}$ (for V/III ratio of 130 ).$^{21}$ By contrast, Joyce et al. ${ }^{11}$ reported that the crystal structure changes from ZB-dominant to WZ-dominant with increasing temperature in the range of $400-500{ }^{\circ} \mathrm{C}$ (for $\mathrm{V} / \mathrm{III}$ ratio of 46). Both studies are performed in metal-organic vapor phase epitaxy (MOVPE) using the same precursors, and both take diameter effects into consideration. Nevertheless, the nanowires are grown in different systems (therefore different temperature calibration, reactor geometry, reactor history, substrate origin and treatment, precursor flows, and so forth are not directly comparable), so it is very difficult to directly infer anything from the apparently opposite behavior observed.

Furthermore, we have found that InAs exhibits a dramatic change in the crystal structure around $400{ }^{\circ} \mathrm{C}$ : nanowires below this temperature are mostly $\mathrm{ZB}$, while above $400{ }^{\circ} \mathrm{C}$ nanowires are mostly $\mathrm{WZ}$, with increasing $\mathrm{ZB}$ contribution as temperature is increased further. ${ }^{22}$ This clearly shows that even for a single accessible parameter, crystal structure variation is not straightforward. In itself this should not be surprising since a variety of processes can influence nanowire growth, including system thermodynamics, nucleation kinetics, precursor decomposition kinetics (in systems using chemical precursors), diffusion of growth species along the substrate and nanowire side facets, and diffusion through the seed particle. Nanowire growth occurs at the interface between the seed particle and the crystal material and therefore should be influenced by the supersaturation and (for III-V materials) V/III ratio at this influence. Since these parameters can be influenced by particle solubility and diffusion coefficients for the different species, surface diffusion coefficients, precursor decomposition, and, of course, the concentrations in the vapor phase, they can depend in nontrivial ways on temperature, pressure, and precursor input flows. ${ }^{22}$

In this work, we investigate the dependence of the crystal structure on the growth parameters for Au particle-seeded InAs nanowires, with the aim of getting a more complete picture of the structural variations within the full multidimensional parameter space. We consider diameter, temperature, precursor flow (for constant V/III ratio), and V/III ratio (varying both group III and group V independently). Such extensive investigation is necessary to fully understand and control the crystal structure of this III-V nanowire material. Moreover, it is critical for the transfer of knowledge between different growth systems and the application of general models to all systems that the variations of all directly accessible growth parameters are understood.

\section{EXPERIMENT}

InAs nanowires have been grown using $\mathrm{Au}$ aerosol seed particles $^{23}$ of selected diameters in the range of $10-110 \mathrm{~nm}$, deposited onto InAs (111)B substrates with surface densities in the range of 1-3 $\mu \mathrm{m}^{-2}$. For diameter-dependence studies, $\mathrm{Au}$ particles with several diameters were deposited onto the same substrates, while for studies of other parameter dependencies, single diameter particles were deposited onto each substrate to achieve better statistics.

Nanowires were grown using low-pressure MOVPE (10 $\mathrm{kPa}$ ) in $13 \mathrm{~L} / \mathrm{min}$ hydrogen flow. The precursors used were trimethylindium (TMI) and arsine $\left(\mathrm{AsH}_{3}\right) ; \mathrm{AsH}_{3}$ was maintained during the entire growth run (including heating/ cooling), while the TMI flow was switched on and off to initiate and end growth. Precursor flows were investigated in the range of $1.4 \times 10^{-6}-2.1 \times 10^{-5}$ for TMI and 1.4 $\times 10^{-4}-1.7 \times 10^{-3}$ for $\mathrm{AsH}_{3}$. Growth temperatures between 370 and $480{ }^{\circ} \mathrm{C}$ were investigated. No annealing step was performed prior to growth, but for growth temperatures below $440{ }^{\circ} \mathrm{C}$, the growth was initiated by a high-temperature nucleation step (typically $3 \mathrm{~min}$ growth at $460{ }^{\circ} \mathrm{C}$, with flows of $6.9 \times 10^{-6}$ for TMI and $4.2 \times 10^{-4}$ for $\mathrm{AsH}_{3}$, after which the TMI was switched off and temperature and flows ramped to the desired values). The high-temperature nucleation step is used to prevent kinking, which can occur for nanowires nucleated directly at lower temperatures, as discussed in Ref. 24.

Initial morphological characterization was performed using a field-emission scanning electron microscope (FEI Nova NanoLab 600) operated at $15 \mathrm{kV}$. Crystal structure characterization was performed using a JEOL-3000F field-emission transmission electron microscope (TEM) operated at $300 \mathrm{kV}$. TEM images were recorded along the $\langle\overline{1} 10\rangle$ zone axis (cubic notation) using a $2 \mathrm{k} \times 2 \mathrm{k}$ CCD camera. Samples for high resolution TEM were obtained by breaking off the nanowires from the substrate and transferring them to lacey carbon-film coated copper TEM grids.

In total, about 130 different sets of growth parameters (temperature, TMI molar fraction, $\mathrm{AsH}_{3}$ molar fraction, diameter, and growth time) were investigated. All of these samples were investigated by TEM, with typically five nanowires being analyzed for each set of growth conditions. An exception was diameter, for which we studied a very broad selection of different sizes rather than multiple nanowires for each of a few diameters.

\section{RESULTS AND DISCUSSION}

\section{A. Qualitative description}

Before describing the details of the parameter space mapping results, some general observations can be made. First, the observed facets on the nanowires are very consistent, with only a few differences over the entire parameter space. 
WZ phase segments in nanowires are always terminated by $\{\overline{1} 100\}$ facets (as reported in literature ${ }^{22}$ ), while ZB segments have $\{111\}$-type facets if they are short, and $\{112\}$-type facets as the ZB section becomes longer than about $10 \mathrm{~nm}$. Pure ZB nanowires appear to have hexagonal cross section with $\{112\}$-type facets at the very tip, but exhibit significant lateral overgrowth away from the tip. It is noteworthy that we observe significantly more lateral overgrowth on nanowire segments of ZB structure, compared to segments of WZ structure. ZB overgrowth occurs preferentially on $\{112\}$ A facets with very little overgrowth visible on the $\{112\} B$ facets, yielding a triangular cross section from about $100 \mathrm{~nm}$ away from the tip. When twin planes occur in ZB nanowires, the rotation of the facets results in alternating triangular segments, as shown by Zou et al. ${ }^{25}$ Aside from occasional inclined twins, lateral overgrowth is observed to always follow the crystal structure of the underlying nanowire.

In order to quantify the effects of temperature, V/III ratio, diameter, and precursor flow on the InAs nanowire crystal structure, we need to select a convention for assigning a value to the observed structure. However, the assignment of a single number to the structure obscures qualitative differences. To clarify this, we note that there are actually two variables of interest here: the proportion of each phase (ZB and WZ) and the density of stacking defects or twin defects. Although these variables are related, they are not directly interchangeable.

There are two common ways to define the proportion of different phases in nanowires. In the first method, individual planes are determined to be of cubic or hexagonal ( $\mathrm{c}$ or $\mathrm{h}$ ) type, and the total c- and h-planes are added up without consideration for their sequence. In the second method, planes are assigned to a specific phase (ZB or WZ) depending on how many occur in a row: ZB phase requires at least two consecutive c-planes, while $\mathrm{WZ}$ requires at least two consecutive h-planes. In this case, single planes are counted only as defects and may be tabulated to compute defect density-but are not counted in the proportion of the secondary phase.

The difference in these conventions arises from the consideration of long-range order (or the lack of it) in nanowire stacking. Nanowires are currently understood to grow layer-by-layer, ${ }^{26}$ and kinetic models typically focus on the probability of a single layer irrespective of the structure of the underlying layers. ${ }^{13}$ In this way, the probability of forming two c-planes in sequence is identical to the probability of forming two c-planes very separated from each other, and their distribution is governed only by statistics. These models often fit very well with experimental observations, but they have difficulty explaining nanowire structures grown under constant conditions which exhibit extended segments $(>5$ sequential bilayers) of both $\mathrm{WZ}$ and $\mathrm{ZB}$ throughout the nanowire. When long-range order is considered, it becomes important to consider extended segments of WZ and ZB explicitly (rather than simply c and h stacking).

We must also consider the effect of segment thickness on the electronic structure. While it is clear that a single h-plane

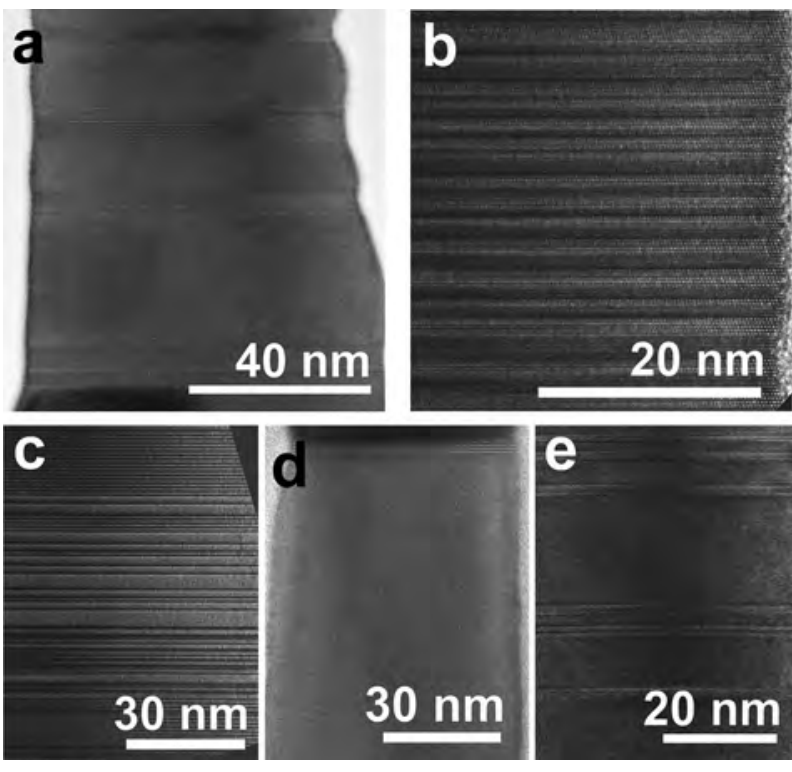

FIG. 1. TEM images showing crystal structure variations in InAs nanowires. (a) Predominantly ZB nanowire with WZ inclusions of several nanometer long. The c-plane proportion for this nanowire is 0.8. (b) Predominantly ZB nanowire with a high density of individual twin planes. The c-plane proportion for this nanowire is 0.8 . (c) Nanowire containing a mixture of ZB and WZ phases with randomly distributed stacking faults and twin planes. The c-plane proportion is close to 0.5. (d) Pure ZB nanowire with c-plane proportion of 1, with the exception of a single WZ segment near the top interface ("neck region"). (e) Predominantly ZB nanowire containing twin planes, arranged in pairs such that there is a favored twin segment orientation. The c-plane proportion is 0.97 .

within an otherwise ZB nanowire will not have the same electronic structure as a purely $\mathrm{WZ}$ nanowire, it is not clear how long a WZ segment needs to be before its electronic structure is identical to an infinitely long segment. Consideration of typical segment lengths is therefore important for understanding measurements. Also, we have noted above that single defect planes that change the stacking sequence can act as electron scattering centers. ${ }^{6,7}$ It is therefore most convenient to consider only the density of defects/interfaces to understand electron transport measurements. Indeed, whenever nanowires can be characterized by a common parent phase containing single defect planes, defect density is the most convenient notation regardless of the purpose of the study.

However, none of the above notations (proportion of cand h-planes, proportion of WZ and ZB phase, and density of stacking defects) is sufficient in itself to fully describe the structure when the crystal structure is mixed. We note first that the nanowires shown in the following images [Figs. 1(a) and $1(\mathrm{~b})]$ have the same relative $\mathrm{c}-$ and h-plane density. In the first case, the nanowire is predominantly ZB with $\mathrm{WZ}$ inclusions of $5-10 \mathrm{~nm}$ in length. In the second case, the nanowire is predominantly $\mathrm{ZB}$ with frequent single $\mathrm{h}$-planes (twin planes). Both nanowires have a c-plane proportion of about 0.8 , but they are obviously very different materials and may have different properties. In the first case, all ZB portions have the same orientation, while in the second case there is a random mixing of two twin orientations. Also, very 
different density of structural interfaces will cause significantly different electron scattering, while band bending at interfaces will also give rather different electronic band diagrams.

On the other hand, if we consider only extended WZ and ZB phase, nanowires containing only isolated single defect planes are not considered to have a mixed phase. The nanowire in Fig. 1(b) (above), which has only single h-planes separated by consecutive c-planes, has no extended WZ phase and might therefore be considered entirely ZB. However, it is obviously very different from the nanowire shown in Fig. 1(c), which is defect-free ZB phase. In this situation, or the alternative case where most nanowires are $\mathrm{WZ}$ with only single stacking faults, the most useful parameter would be defect density. Defect density alone, on the other hand, cannot be used to compare samples with nanowires of predominantly different phases [it would not, for example, have meaning for Fig. 1(a) (above)] since the definition of a defect plane depends on the parent phase.

Finally, we note one further case of predominantly ZB nanowires, which are qualitatively different from the other nanowires shown in Fig. 1. The nanowire shown in Fig. 1(e) has predominantly ZB structure, with h-planes representing only 0.03 of the structure. All h-planes appear as twin planes, with no stacking faults or extended WZ segments. What is notable in this structure is that twin planes always appear in pairs, so there is a preferred twin orientation for the entire nanowire. The structure of this type has been reported before $^{25,27}$ and we observe in this study that there is, in fact, a preferred twin orientation for all nanowires with nearly pure ZB structure.

It is clear that no single parameter can adequately describe the structure of nanowires such as the one shown in Fig. 1(c), which has a mixture of ZB and WZ segments of various lengths, as well as single stacking defects within the phases. For the purpose of this study, we will quantify structure using the notation of c- and h-planes; the label "ZB proportion" in the axis of all graphs is a measure of the proportion of c-planes. However, whenever possible we will also discuss the qualitative changes in structure and illustrate this with images.

\section{B. Temperature and diameter effects}

We first consider the effect of diameter on the crystal structure for nanowires grown with different temperatures. We have previously reported that nanowires grown in the temperature range of $420-480{ }^{\circ} \mathrm{C}$ show an increase in ZB proportion with increasing diameter for all temperatures. ${ }^{28}$ Moreover, the proportion of $\mathrm{ZB}$ increases with temperature in this range. This effect was explained by a model incorporating the Gibbs-Thomson effect on the supersaturation in the alloy seed particle. We note that diameter effects on supersaturation at the particle-nanowire interface may also occur if nanowire growth is strongly influenced by surface diffusion, and such effects, in principle, could affect the crystal structure. ${ }^{29}$ However, the predictions of this model did not fit the current data and thus are not considered further in this

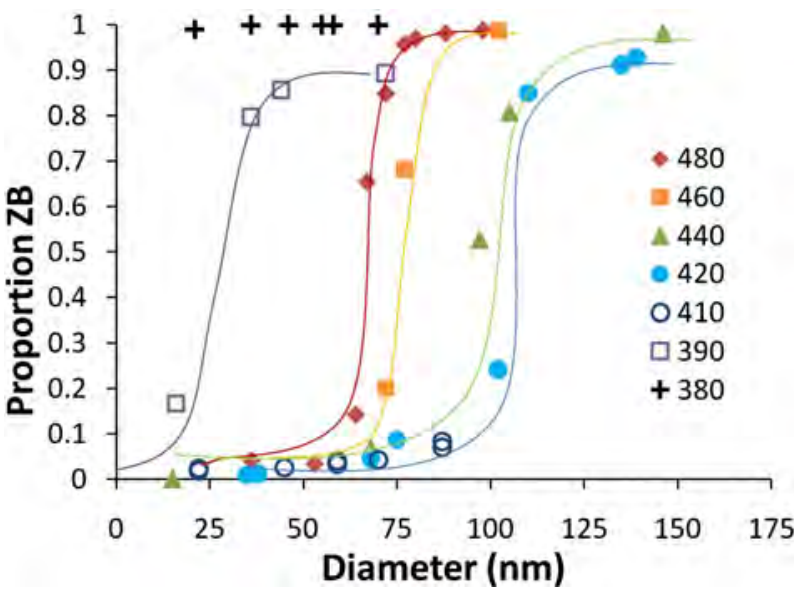

FIG. 2. (Color online) Proportion of ZB phase (counted as proportion of c-planes) vs nanowire diameter for different growth temperatures, at a V/III ratio of $100\left(380-410{ }^{\circ} \mathrm{C}\right)$ and $130\left(420-480{ }^{\circ} \mathrm{C}\right)$, and TMI molar fraction of $6.9 \times 10^{-6}$. Lines are guides for the eye. For all temperatures, there appears to be a transition from $\mathrm{WZ}$ to $\mathrm{ZB}$ with increasing diameter, such that there is a characteristic transition diameter for each temperature. In the temperature range of $410-480^{\circ} \mathrm{C}$, the transition diameter decreases with temperature, but for temperatures below $400{ }^{\circ} \mathrm{C}$, the transition is shifted to a much lower temperature (such that no transition is visible for $380{ }^{\circ} \mathrm{C}$ within the investigated diameter range).

work. In the present work, we extend the earlier investigation of diameter to include lower temperatures; Fig. 2 shows the diameter dependence of the crystal structure of nanowires grown at 410,390 , and $380{ }^{\circ} \mathrm{C}$, presented together with the previously published data ${ }^{28}$ in the temperature range of $420-480{ }^{\circ} \mathrm{C}$.

The data in Fig. 2 are presented for similar V/III ratios $\left(\mathrm{V} / \mathrm{III}=130\right.$ for $420-480{ }^{\circ} \mathrm{C}$ and $\mathrm{V} / \mathrm{III}=100$ for $380-410^{\circ} \mathrm{C}$ ). Since we did not observe a change in diameter dependence for V/III ratio over 50, up to at least 220, the data for which the best statistics are available are presented in this figure. However, if we consider a much lower V/III ratio and look more closely at the transition region, the picture becomes more complicated. Figure 3 shows the diameter dependence of structure for nanowires grown at $410{ }^{\circ} \mathrm{C}$ and V/III 20. Surprisingly, there is a clear increase in the proportion of ZB phase with decreasing diameter. The data for the same temperature and V/III ratio100 are included for comparison.

Although the available data are not sufficient to investigate the origin of this, it may be that the temperature at which the switch from ZB to WZ occurs shifts with V/III ratio and diameter. For most of the parameters investigated in this study, there is a minimum in ZB proportion at temperature of $410^{\circ} \mathrm{C}$, as shown in Fig. 4. However, this minimum may shift toward $420^{\circ} \mathrm{C}$ for very low V/III ratio and certain diameters. It may also shift toward $400{ }^{\circ} \mathrm{C}$ for other parameters: we observed a small but statistically significant increase in $\mathrm{WZ}$ purity when reducing the temperature from 410 to $400{ }^{\circ} \mathrm{C}$ for V/III 90 when precursor molar fractions were halved relative to those in Fig. 2.

Returning to the temperature dependence of the data presented in Fig. 2, we note that the crystal structure of nano- 


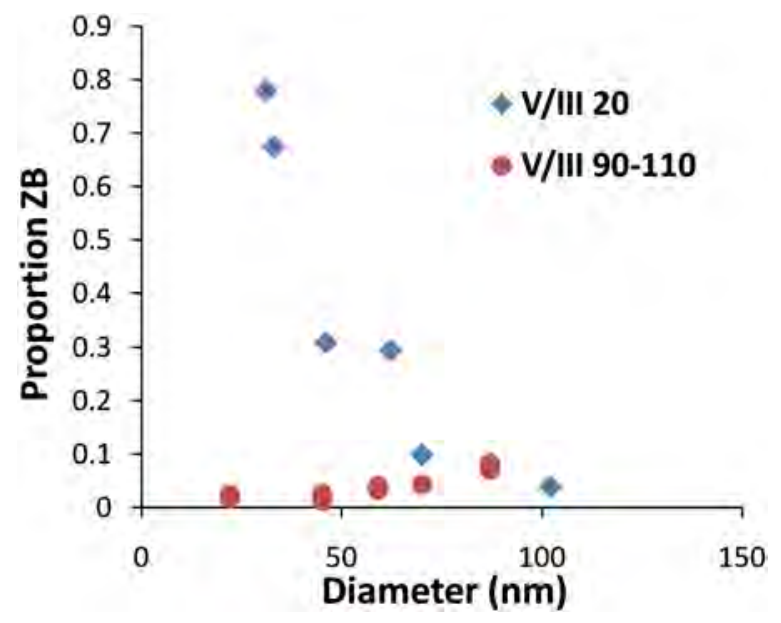

Fig. 3. (Color online) Proportion of ZB phase vs nanowire diameter for growth temperature of $410^{\circ} \mathrm{C}$ and TMI molar fraction of $6.9 \times 10^{-6}$, at $\mathrm{V} / \mathrm{III}$ ratios of 20 and 100 (90-110 plotted together). There is a clear difference in the dependence, with ZB proportion increasing with diameter at high $\mathrm{V} / \mathrm{III}$, but decreasing with diameter at low V/III.

wires grown at 380 and $390{ }^{\circ} \mathrm{C}$ is entirely different from the other data presented. While the transition diameter from WZ to $\mathrm{ZB}$ increases with temperature in the range of $410-480{ }^{\circ} \mathrm{C}$, the transition diameter at $390{ }^{\circ} \mathrm{C}$ is much smaller (the curve is shifted toward smaller diameters), indicating that nanowires at this temperature will be predominantly ZB for most diameters. At a temperature of $380{ }^{\circ} \mathrm{C}$, the data indicate that nanowires are pure $\mathrm{ZB}$ for all diameters, likely indicated that the transition diameter is below the range investigated. We have also reported previously ${ }^{22}$ that there is an abrupt transition from pure or nearly pure ZB nanowires at temperatures below $400{ }^{\circ} \mathrm{C}$ to pure or nearly pure $\mathrm{WZ}$ at temperatures above $400{ }^{\circ} \mathrm{C}$. Here, we look more closely at this transition by investigating the temperature dependence of the crystal structure for specific diameters and V/III ratio, as presented in Fig. 4.

\section{V/III ratio effects}

When considering the effects of V/III ratio and total mass flow (discussed in Sec. III D), it is important to recall that we are referring to the input ratio/flows of the precursor species, which are directly accessible parameters. However, these parameters do not directly correlate to V/III ratio and supersaturation at the nanowire growth front, which are determined by system thermodynamics and kinetics. ${ }^{22}$ Therefore, they may influence growth and crystal structure in complicated ways. The temperature dependence of the nanowire crystal structure is presented in Fig. 4 on a log scale; we can see that there is, in fact, a minimum in $\mathrm{ZB}$ proportion with temperature around $410{ }^{\circ} \mathrm{C}$. As temperature is decreased below this, nanowires become increasingly $\mathrm{ZB}$, and as temperature is increased above $410{ }^{\circ} \mathrm{C}$, the density of stacking faults in WZ increases with a tendency to form ZB phase as the temperature approaches $480{ }^{\circ} \mathrm{C}$. To investigate further, we select the temperatures of 380 and $410{ }^{\circ} \mathrm{C}$ since these two temperatures tend to give nanowires with predominantly

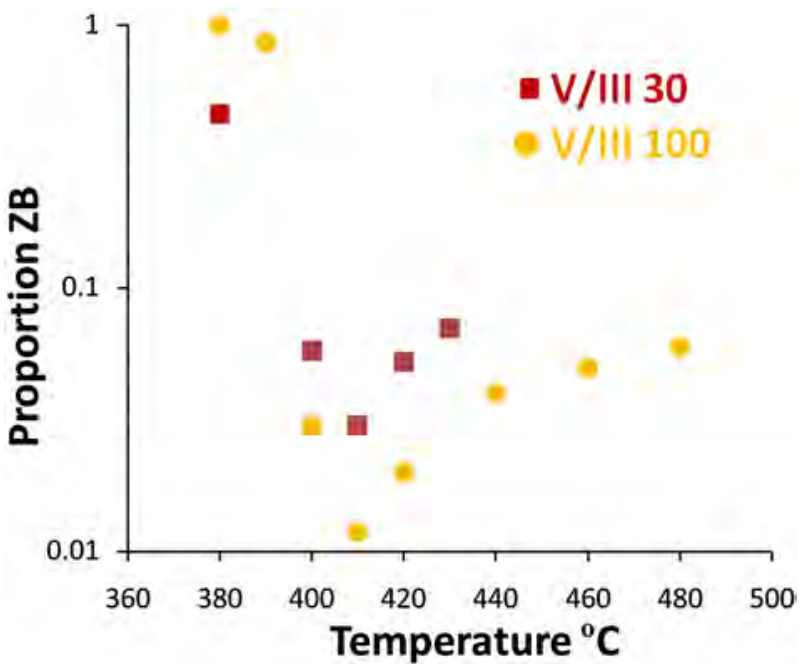

FIG. 4. (Color online) Proportion of ZB phase vs growth temperature for nanowires grown with TMI molar fraction of $6.9 \times 10^{-6}$ and V/III ratios of 30 and 100. All nanowires have a diameter of $45 \pm 2 \mathrm{~nm}$. There is a sharp transition from predominantly ZB to predominantly WZ phase at a temperature around $410^{\circ} \mathrm{C}$. Above this temperature, the proportion of $\mathrm{ZB}$ decreases slowly. The transition is sharper for higher V/III ratio.

$\mathrm{ZB}$ and predominantly $\mathrm{WZ}$ phase, respectively. V/III ratio investigations are performed by varying $\mathrm{AsH}_{3}$ flow unless otherwise noted; TMI molar fractions were fixed to 6.9 $\times 10^{-6}$ for both temperatures.

We observe that the crystal structure is highly sensitive to V/III ratio. At high V/III ratio (above about 80-90), nanowires grown at $380^{\circ} \mathrm{C}$ are pure $\mathrm{ZB}$ without stacking defects. We have observed that there is no variation in structure for $\mathrm{V} / \mathrm{III}$ ratio from 80 to 240 (pure $\mathrm{ZB}$ in all cases). There is also no significant difference in structure for V/III ratios in the range of $50-224$ at $410{ }^{\circ} \mathrm{C}$ for all diameters. In other words, the transition from pure $\mathrm{ZB}$ to pure $\mathrm{WZ}$ with increasing temperature is very sharp, and there is no obvious region of mixed structure.

At lower V/III ratios, the transition from ZB phase to $\mathrm{WZ}$ phase with temperature is observed to be less sharp. At V/III ratio of $30, \mathrm{ZB}$ structure still dominates at the lowest temperatures, but the structure is no longer pure: twin planes are present with density increasing directly with temperature. At $400{ }^{\circ} \mathrm{C}$, the nanowires are predominantly WZ, but with relatively dense stacking faults. A minimum in stacking fault density is observed at $410{ }^{\circ} \mathrm{C}$, but even here, the structure is significantly less pure at low V/III ratio than at high V/III ratio. Stacking fault density increases with temperature as usual. The temperature behavior is therefore qualitatively similar to that at higher V/III ratio, but the transition is much less sharp and pure phases are not observed (Fig. 4).

Decreasing V/III ratio further, to 20 at a temperature of $410{ }^{\circ} \mathrm{C}$, we observe significant $\mathrm{ZB}$ inclusions in the predominantly $\mathrm{WZ}$ nanowire, with the $\mathrm{WZ}$ proportion dropping below 0.7. Once again, this indicates that the lowtemperature ZB-WZ transition is smoothed out for low V/III ratio. We note that this is clearly linked to the unusual diameter dependence illustrated in Fig. 3; at large diameters, there 


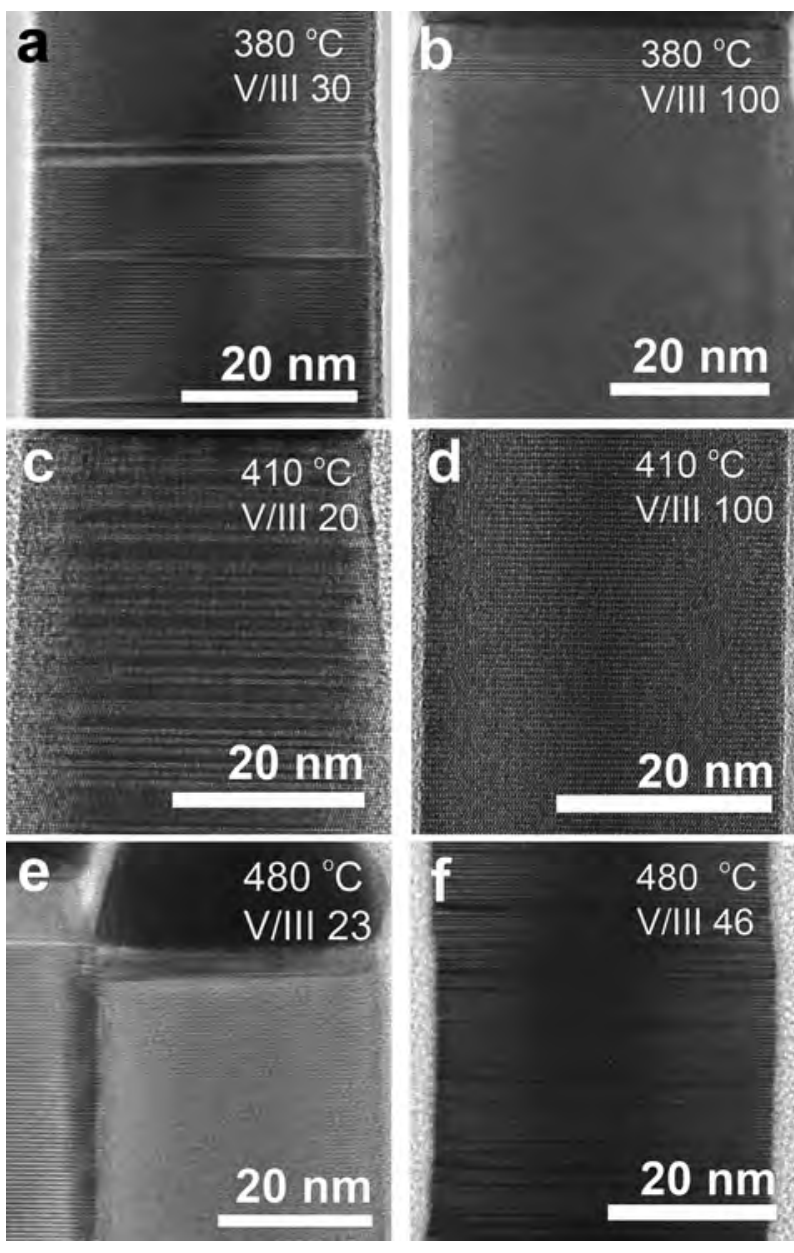

FIG. 5. TEM images illustrating the effect of V/III ratio on InAs nanowire crystal structure at different temperatures. (a) Predominantly WZ nanowires grown at $380^{\circ} \mathrm{C}$, TMI of $6.9 \times 10^{-6}, \mathrm{~V} / \mathrm{III}$ of 30 , diameter of $38 \mathrm{~nm}$, and $\mathrm{ZB}$ proportion of 0.28 . (b) Pure $\mathrm{ZB}$ nanowires grown at $380{ }^{\circ} \mathrm{C}$, TMI of 6.9 $\times 10^{-6}$, V/III of 100 , diameter of $45 \mathrm{~nm}$, and $\mathrm{ZB}$ proportion of 1 . (c) $\mathrm{ZB}$ nanowires with frequent twin planes grown at $410^{\circ} \mathrm{C}$, TMI of $6.9 \times 10^{-6}$, V/III of 20, diameter of $35 \mathrm{~nm}$, and ZB proportion of 0.68. (d) Pure WZ nanowires grown at $410^{\circ} \mathrm{C}$, TMI of $6.9 \times 10^{-6}$, V/III of 100 , diameter of 33 $\mathrm{nm}$, and $\mathrm{ZB}$ proportion of 0 . (e) Pure $\mathrm{WZ}$ nanowires grown at $480{ }^{\circ} \mathrm{C}$, TMI of $6.0 \times 10^{-6}$, V/III of 23 , diameter of $41 \mathrm{~nm}$, and ZB proportion of 0 . (e) WZ nanowires with frequent stacking faults grown at $480{ }^{\circ} \mathrm{C}$, TMI of 6.0 $\times 10^{-6}, \mathrm{~V} / \mathrm{III}$ of 46 , diameter of $39 \mathrm{~nm}$, and $\mathrm{ZB}$ proportion of 0.12 .

is no significant difference in the crystal structure with V/III ratio, but as the diameter decreases, the sensitivity to V/III ratio increases.

Interestingly, we note that the effect of V/III does not appear to be to change the structure from $\mathrm{WZ}$ to $\mathrm{ZB}$ (or vice versa). Rather, increasing V/III ratio seems to cause a transition from mixed structure to pure structure. Turning again to Fig. 4 , we can see that at $380^{\circ} \mathrm{C}$, the proportion of $\mathrm{ZB}$ increases with V/III ratio (toward pure ZB), while at $410{ }^{\circ} \mathrm{C}$, the proportion of $\mathrm{WZ}$ increases with $\mathrm{V} / \mathrm{III}$ ratio (toward pure WZ). This is also illustrated in Figs. 5(a)-5(d). In other words, the effect of V/III ratio seems to be to abruptly reduce the density of stacking defects in the dominant phase at a certain critical value (around V/III of 100 for temperature of $380{ }^{\circ} \mathrm{C}$, around 50 for temperature of $410{ }^{\circ} \mathrm{C}$, at TMI of $\left.6.9 \times 10^{-6}\right)$. Above these critical ratios, we observe no
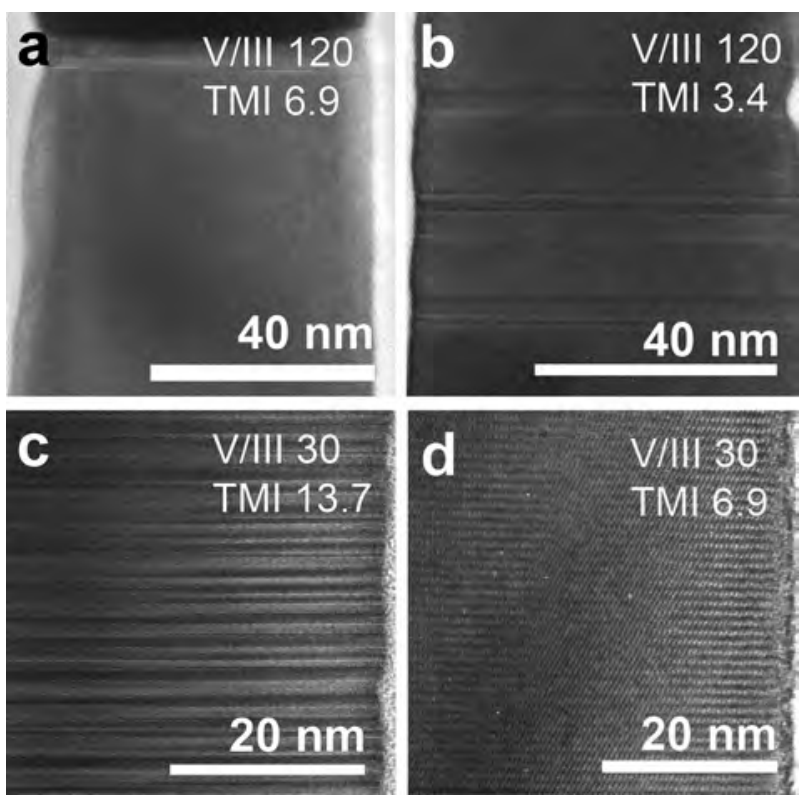

FIG. 6. TEM images illustrating the effect of precursor mass flow on InAs nanowire crystal structure for selected V/III ratios; TMI flows indicated on the images are $\times 10^{-6}$. Nanowires are grown at $380^{\circ} \mathrm{C}$. (a) Pure ZB nanowire grown at V/III of 120 , TMI of $6.9 \times 10^{-6}$, and diameter of $58 \mathrm{~nm}$. (b) $\mathrm{ZB}$ nanowire with $\mathrm{WZ}$ segment inclusions of several $\mathrm{nm}$ in length, grown at $\mathrm{V} / \mathrm{III}$ of 120 , TMI of $3.4 \times 10^{-6}$, and diameter of $61 \mathrm{~nm}$. (c) ZB nanowire with frequent twin planes grown at V/III of 30 and TMI of $1.37 \times 10^{-5}$. (d) WZ nanowire with occasional stacking faults grown at V/III of 30 and TMI of $6.9 \times 10^{-6}$.

change in the structure with V/III ratio, even at $410{ }^{\circ} \mathrm{C}$ where the predominant phase (WZ) does not make up 100\% of the structure.

Furthermore, we observe that the effect of V/III ratio appears to be independent of the total mass flow and therefore not obviously an effect of supersaturation within the seed particle. Referring again to Figs. 5(a) and 5(b), we note that the change in V/III ratio is achieved by increasing the $\mathrm{AsH}_{3}$ while keeping the TMI constant. If we instead lower the TMI while keeping the $\mathrm{AsH}_{3}$ constant, we again observe a change from mostly WZ to mostly ZB phase. This is shown in Figs. 6(a) and 6(b) - in the former, the TMI is the same as in Fig. 5(a), while the $\mathrm{AsH}_{3}$ is higher, and in the latter, the TMI is lower than in Fig. 5(a), while the $\mathrm{AsH}_{3}$ is the same. Although there are qualitative differences between these samples (as discussed in Sec. III D), both are clearly ZB-dominant while the sample in Fig. 5(a) is WZ-dominant.

To further complicate the picture, we have also considered the effect of V/III ratio on nanowires grown at $480{ }^{\circ} \mathrm{C}$. We have previously reported that there is negligible variation of stacking fault density in nanowires grown with V/III ratios from 46 to $1500 .^{30}$ However, we observe that when the V/III ratio is lowered further to 23 at this temperature, the structure changes abruptly to pure WZ [Figs. 5(e) and 5(f)]. These results indicate that the apparently contradictory results of Caroff et al. and Joyce et al. mentioned in Sec. I may be entirely explained by V/III ratio. For a V/III ratio around 20, we observe primarily $\mathrm{ZB}$ at $410{ }^{\circ} \mathrm{C}$ and primarily $\mathrm{WZ}$ at $480^{\circ} \mathrm{C}$, entirely opposite to the dependence observed for 


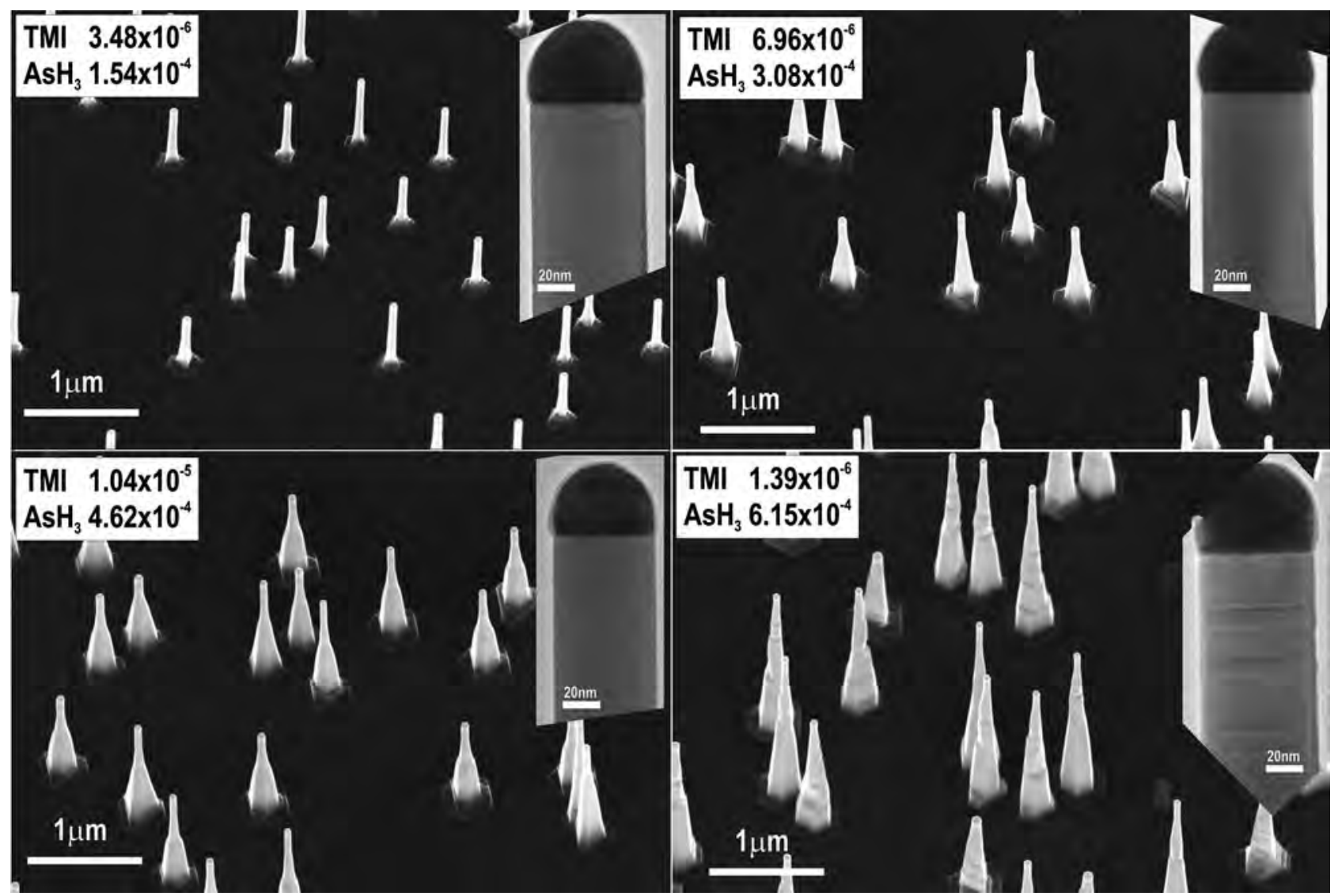

FIG. 7. Images illustrating the effect of precursor mass flow on InAs nanowire growth at a growth temperature of $415{ }^{\circ} \mathrm{C}$ and V/III ratio of 44 . The precursor flows are $3.48 \times 10^{-6}$ for and $1.54 \times 10^{-4}$ for $\mathrm{AsH}_{3}$ multiplied by factors of (a) 1, (b) 2, (c) 3, and (d) 4 . The insets of (a)-(d) are TEM images illustrating the crystal structure for each of the conditions. It is clear that the growth rate and lateral overgrowth scale with the precursor mass flow, but any effect on the crystal structure is minimal.

V/III of 100. Allowing for differences in reactor geometry, temperature calibration, and absolute precursor flows (which preclude direct comparison of parameters), these results appear consistent with both previously reported data sets.

\section{Total mass flow effects}

Up to now, we have considered the variation of the crystal structure with diameter, temperature, and V/III ratio for a constant TMI molar fraction. We have also observed, by varying the TMI and $\mathrm{AsH}_{3}$ flows simultaneously, a dependence of the crystal structure on the total precursor mass flow. In Fig. 6, we show images of nanowires grown at $380{ }^{\circ} \mathrm{C}$ with V/III ratio of $30[(\mathrm{c})$ and (d)] and $120[$ (a) and (b)]. In both cases, doubling the precursor flows leads to a significant increase in the proportion of $\mathrm{ZB}$ in the nanowires, regardless of the initial phase. This consistent change of structure with total mass flow suggests a straightforward supersaturation effect, as predicted by several models. ${ }^{31,32}$ However, all of these models predict a decrease in ZB structure with increasing supersaturation (toward WZ phase). The results here, on the other hand, are consistent with the observation of Joyce et al. for GaAs, that ZB phase purity increases with total mass flow. ${ }^{15}$
In addition, here again the change in structure appears abruptly, over a narrow range of flows, and further increase or decrease of these flows has little effect on the structure. We observe, for example, for nanowires grown at $415^{\circ} \mathrm{C}$ and V/III 44, increasing the total precursor flows by a factor of 4 results in an approximately fourfold increase in growth rate [Figs. $7(\mathrm{a})-7(\mathrm{~d})]$, indicating that supersaturation is indeed increased directly as expected. However, there is no significant change in the crystal structure, which is primarily WZ with moderate-density stacking faults [Figs. 7(a)-7(d), insets]. The same trend is observed at $480{ }^{\circ} \mathrm{C}$ : for V/III ratios above the transition shown above, there is no change in the crystal structure with total mass flow in the entire range achievable for our growth system.

Finally, we note that care must be taken when comparing nanowire crystal phase to account for any variations of structure with length. There are a few reports of nanowire crystal structure changing with distance from the substrate, ${ }^{18,28}$ attributed to the decrease in precursor collection area (and therefore mass flow) as the nanowire length approaches the precursor surface diffusion length. We have previously noted that nanowire growth rate becomes constant only after about $1 \mu \mathrm{m}$ at a growth temperature of $380{ }^{\circ} \mathrm{C}$ (indicating that nanowire length exceeds the diffusion length and therefore 
supersaturation is length-independent). ${ }^{33}$ In this work, we have also observed small changes in the $\mathrm{ZB}$ proportion of nanowires grown at $415{ }^{\circ} \mathrm{C}$ : for low $\mathrm{AsH}_{3}$ flows, there is an increase in stacking defects within $\mathrm{WZ}$ phase in the lower region of the nanowires, while at high $\mathrm{AsH}_{3}$ flows, there is an increase in stacking defects in WZ phase in the upper part of the nanowires, for a range of V/III ratios.

\section{E. Relationship to current models}

An important observation in this work is that transitions in crystal structure with growth parameters are not necessarily gradual, as would be anticipated by current models based on nucleation kinetics. Rather, we observe that structure can change very significantly with a very small change of temperature, mass flow, or V/III ratio, while subsequently changing very little over parameter changes of an order of magnitude or more. While this is not directly predicted by current nucleation models, it is not necessarily inconsistent with them, if input parameters change at critical conditions. These abrupt shifts in crystal structure imply a thermodynamic effect on structure, which may relate to changes in surface reconstructions or alloy particle phase/composition. ${ }^{34}$ Indeed, it is well-known that surface reconstructions of III-V semiconductors can be affected by the input group V flow. ${ }^{11}$ Incorporating these effects directly into future theoretical models may greatly enhance their relevance and application.

A second important result from this work is the observation that V/III ratio plays a critical role in crystal structure, changing the structure entirely at a certain critical ratio. Moreover, the direction of this structural change depends on temperature, diameter, and most likely precursor mass flow. This sensitivity of structure to V/III ratio is consistent with reports in other systems, ${ }^{15,20-22}$ but is not accounted for by current models. Most theoretical models focusing on nucleation kinetics explain changes in structure with temperature, supersaturation, and diameter. Here, "supersaturation" often refers to an overall supersaturation (presumably including both precursors) or explicitly refers to supersaturation of the group III species (which alloys with the seed particle in measurable quantities). The group V species has, in most cases, been ignored in theoretical calculations. A recent model by Glas suggests that the group V species must also be considered and gives predictions of the effect of this species on crystal structure. ${ }^{35}$ However, this model still essentially focuses on total supersaturation. Therefore, a decrease in group III species, for example (decreased supersaturation, increased V/III ratio), will have the opposite effect on the crystal structure compared to an increase in group $\mathrm{V}$ species (increased supersaturation, increased V/III ratio). The results in this article clearly suggest that V/III ratio cannot be directly related to supersaturation, but rather must be treated as an important and distinct parameter in future theoretical models.

\section{F. Role of precursor chemistry}

In order to gain insight into the relationship of crystal phase to V/III ratio and total supersaturation, it is necessary to consider how the tunable parameters are connected to real growth parameters in MOVPE. Most III-V nanowires, including those in this study, are grown at low temperatures compared to the temperatures used for conventional layer growth by MOVPE. ${ }^{36}$ The decomposition of the precursor molecules depends strongly on the temperature, especially in the temperature range usually used for NW growth. It has been shown in pyrolysis experiments that the decomposition of the precursors can be greatly enhanced when both hydride and metal-organic precursor molecules are present simultaneously. This has been shown for trimethylindium (TMI) + phosphine $\left(\mathrm{PH}_{3}\right)$ (Ref. 37) and trimethylgallium (TMG) + arsine $\left(\mathrm{AsH}_{3}\right){ }^{38}$ indicating that this is probably also the case for other combinations of these precursors such as TMI and $\mathrm{AsH}_{3}$. In addition, in the same studies, it was shown that the substrate surface strongly influences the amount of decomposed species (linear dependence on substrate surface area), which lead to the conclusion that at low temperature the pyrolysis is mainly heterogeneous. In both studies, it was found that the hydrides, in particular, start to decompose at significantly lower temperatures in the presence of the metalorganic molecules.

These results clearly show that the decomposition of the metal-organic and the hydride molecules are probably strongly connected during NW growth as well. A very important conclusion from the studies in Refs. 37 and 38 is that at low temperatures, because the decomposition of the hydride and the metal-organic are characterized by a joint pyrolysis reaction, the actual V/III ratio of the decomposed species is unity. This means that increasing either the $\mathrm{V}$ or the III molar flow/supply will not change the true V/III ratio-it can only increase the amount of both. Normally, the input V/III ratio is larger than 1 (group $\mathrm{V}$ precursor is in excess) during MOVPE growth of III-V materials. This suggests that under certain conditions, increasing the molar flow of group $\mathrm{V}$ precursor should not affect the growth, but increasing group III would lead to more III and V molecules being decomposed and thus increase the growth rate. However, this does not account for the fact that significant amounts of TMI may also decompose independently at nanowire growth temperatures, leading to a competing reaction pathway which may reduce the decomposition efficiency of the $\mathrm{AsH}_{3}$ molecules. This should lead to much more complicated dependencies on precursor flows.

It should also be pointed out that the substrate surface itself influences the reactions taking place, including the decomposition and the diffusion of both the precursor molecules and the decomposed species. This means that the detailed arrangement of the atoms in the surface, which may vary with parameters such as temperature, pressure, and partial pressure of different species present, can influence the growth in a nonintuitive way. The surface coverage of adsorbed molecules of each species and their diffusion on the surface can then strongly influence the rate of decomposition of both types of precursors.

The purpose of this discussion is to illustrate the challenge in connecting the input values of the precursor molar fractions and V/III ratio to the observed dependencies of 
crystal phase on these parameters. It is clear, for example, that changing the V/III ratio could change the total supersaturation of both growth species (In and As), perhaps by a larger magnitude than can be achieved simply by changing the total mass flow of the two precursors simultaneously. However, the dependency would likely be nonlinear, with an optimum V/III ratio (determined by processes including competing chemical reactions, precursor surface adsorption and diffusion, and adatom surface diffusion) for which the ratio of decomposed species would be closest to unity. Since the relevant kinetic processes will all depend on temperature in different ways, one might expect complicated variations of $\mathrm{V} / \mathrm{III}$ ratio dependence for different temperatures—exactly as has been observed in this study.

\section{SUMMARY AND CONCLUSIONS}

In this study, we have investigated the crystal structure of InAs nanowires over a broad parameter space covering temperature, diameter, V/III ratio, and total mass flow. The intention has been to work toward a crystal structural map, to aid in comparing and transferring growth results between different experimental setups and in understanding the trends in polytypism typically observed in this material system. This understanding, in turn, should lead to greater possibilities for structural control suitable for advanced applications. We observe that InAs crystal structure can indeed be tuned between pure or nearly pure ZB and WZ, with a variety of mixtures attainable in between.

We have determined in this study that precursor V/III ratio is an important parameter, the effect of which cannot adequately be understood by considering only supersaturation of the growth species. The reason for this effect is unclear, but may relate to the decomposition chemistry of the metal-organic precursors used. We also observe several cases of "threshold effects" in crystal structure dependence on temperature, V/III ratio, and total mass flow, in which the structure changes significantly at a critical value, while subsequently changing very little over further parameter changes. These abrupt changes indicate that thermodynamic effects may play an important role in determining nanowire structure and should be incorporated into future theoretical models.

\section{ACKNOWLEDGMENTS}

This work was supported by the Nanometer Structure Consortium at Lund University (nmC@LU), the Swedish Foundation for Strategic Research (SSF), the Swedish Research Council (VR), and the Knut and Alice Wallenberg Foundation.

${ }^{1}$ M. Pierre, R. Wacquez, X. Jehl, M. Sanquer, M. Vinet, and O. Cueto, Nat. Nanotechnol. 5, 133 (2010).

${ }^{2}$ J. N. Yang, G. W. Neudeck, and J. P. Denton, J. Appl. Phys. 91, 420 (2002).

${ }^{3}$ Z. Zanolli, F. Fuchs, J. Furthmueller, U. von Barth, and F. Bechstedt,
Phys. Rev. B 75, 245121 (2007).

${ }^{4}$ M. Murayama and T. Nakayama, Phys. Rev. B 49, 4710 (1994).

${ }^{5}$ A. De and C. E. Pryor, Phys. Rev. B 81, 155210 (2010).

${ }^{6}$ Z. Ikonić, G. P. Srivastava, and J. C. Inkson, Phys. Rev. B 48, 17181 (1993).

${ }^{7}$ M. D. Stiles and D. R. Hamann, Phys. Rev. B 41, 5280 (1990).

${ }^{8}$ D. L. Dheeraj, G. Patriarche, H. L. Zhou, T. B. Hoang, A. F. Moses, S. Gronsberg, A. T. J. van Helvoort, B. O. Fimland, and H. Weman, Nano Lett. 8, 4459 (2008).

${ }^{9}$ S. O. Mariager, C. B. Sorensen, M. Aagesen, J. Nygard, R. Feidenhans'1, and P. R. Willmott, Appl. Phys. Lett. 91, 083106 (2007).

${ }^{10}$ K. A. Dick, C. Thelander, L. Samuelson, and P. Caroff, Nano Lett. 10, 3494 (2010).

${ }^{11}$ H. J. Joyce, J. Wong-Leung, Q. Gao, H. H. Tan, and C. Jagadish, Nano Lett. 10, 908 (2010).

${ }^{12} \mathrm{P}$. Caroff, J. Bolinsson, and J. Johansson, IEEE J. Sel. Top. Quantum Electron.

${ }^{13}$ J. Johansson, L. S. Karlsson, C. P. T. Svensson, T. Mårtensson, B. A. Wacaser, K. Deppert, L. Samuelson, and W. Seifert, Nature Mater. 5, 574 (2006).

${ }^{14}$ M. Tchernycheva, J. C. Harmand, G. Patriarche, L. Travers, and G. E. Cirlin, Nanotechnology 17, 4025 (2006).

${ }^{15}$ H. J. Joyce et al., Nano Lett. 9, 695 (2009).

${ }^{16}$ H. J. Joyce et al., Adv. Funct. Mater. 18, 3794 (2008).

${ }^{17}$ H. Shtrikman, R. Popovitz-Biro, A. Kretinin, L. Houben, M. Heiblum, M. Bukala, M. Galicka, R. Buczko, and P. Kacman, Nano Lett. 9, 1506 (2009).

${ }^{18}$ D. M. Cornet, V. G. M. Mazzetti, and R. R. LaPierre, Appl. Phys. Lett. 90, 013116 (2007).

${ }^{19}$ R. E. Algra, M. A. Verheijen, M. T. Borgström, L.-F. Feiner, G. Immink, W. J. P. van Enckevort, E. Vlieg, and E. P. A. M. Bakkers, Nature (London) 456, 369 (2008).

${ }^{20}$ S. Paiman et al., Nanotechnology 20, 225606 (2009).

${ }^{21}$ P. Caroff, K. A. Dick, J. Johansson, M. E. Messing, K. Deppert, and L. Samuelson, Nat. Nanotechnol. 4, 50 (2009).

${ }^{22}$ K. A. Dick, P. Caroff, J. Bolinsson, M. E. Messing, J. Johansson, K. Deppert, L. R. Wallenberg, and L. Samuelson, Semicond. Sci. Technol. 25, 024009 (2010).

${ }^{23}$ M. E. Messing, K. Hillerich, J. Johansson, K. Deppert, and K. A. Dick, Gold Bull. 42, 172 (2009).

${ }^{24}$ H. J. Joyce, Q. Gao, H. H. Tan, C. Jagadish, Y. Kim, X. Zhang, Y. Guo, and J. Zou, Nano Lett. 7, 921 (2007).

${ }^{25}$ J. Zou et al., Small 3, 389 (2007).

${ }^{26}$ F. Glas, J. C. Harmand, and G. Patriarche, Phys. Rev. Lett. 99, 146101 (2007).

${ }^{27}$ R. E. Algra, M. A. Verheijen, L.-F. Feiner, G. G. W. Immink, R. Theissmann, W. J. P. v. Enckevort, E. Vlieg, and E. P. A. M. Bakkers, Nano Lett. 10, 2349 (2010).

${ }^{28}$ J. Johansson, K. A. Dick, P. Caroff, M. E. Messing, J. Bolinsson, K. Deppert, and L. Samuelson, J. Phys. Chem. C 114, 3837 (2010).

${ }^{29}$ J. Johansson, C. P. T. Svensson, T. Mårtensson, L. Samuelson, and W. Seifert, J. Phys. Chem. B 109, 13567 (2005).

${ }^{30}$ C. Thelander, K. A. Dick, M. T. Borgström, L. E. Fröberg, P. Caroff, H. A. Nilsson, and L. Samuelson, Nanotechnology 21, 205703 (2010).

${ }^{31}$ J. Johansson, L. S. Karlsson, K. A. Dick, J. Bolinsson, B. A. Wacaser, K. Deppert, and L. Samuelson, Cryst. Growth Des. 9, 766 (2009).

${ }^{32}$ V. G. Dubrovskii, N. V. Sibirev, J. C. Harmand, and F. Glas, Phys. Rev. B 78, 235301 (2008).

${ }^{33}$ J. Bolinsson, P. Caroff, B. Mandl, and K. A. Dick, Nanotechnology 22, 265606 (2011).

${ }^{34}$ R. E. Algra et al., Nano Lett. 11, 44 (2011).

${ }^{35}$ F. Glas, J. Appl. Phys. 108, 073506 (2010).

${ }^{36}$ G. B. Stringfellow, Organometallic Vapor-Phase Epitaxy: Theory and Practice (Academic, New York, 1999), pp. 324-339.

${ }^{37}$ N. I. Buchan, C. A. Larsen, and G. B. Stringfellow, J. Cryst. Growth 92, 605 (1988).

${ }^{38}$ C. A. Larsen, S. H. Li, N. I. Buchan, G. B. Stringfellow, and D. W. Brown, J. Cryst. Growth 102, 126 (1990). 